\title{
Foucault et la vérité cynique
}

\author{
Foucault e a verdade cínica
}

\section{Frédéric Gros}

Docteur en Philosophie de l'Ecole Normale Supérieure, professeur à l'Université Paris Est Créteil, membre du Centre Michel Foucault, Rédacteur du cours durent par Michel Foucault au Collège de France, Paris - France, e-mail: fredu@club-internet.fr

\section{Résumé}

Synthetiser et développer une série de propositions théoriques à partir des cyniques, travaillées par Foucault dans le cours de 1984, Le courage de la vérité, c'est le but de cet article. Quatre "valeurs" généraux de la pensée grecque y sont distinguées: non-dissimulation, la simplicité, la rectitude et l'immuabilité - valeurs qui ont pris des formes differentes dans trois domaines tradionnels de réflexion: logos, eros, bios. Mais, une autre division, formée au troisième domaine, alêthês bios, a mérité plus d'attention du philosophe. C'est là qu'on observe deux différentes destinées de la vérité. D'un côté, la vie philosophique qui inclut surtout les platoniciens, les epicuriens et les stoïciens. De I'autre, I' "existence" cynique, "sa réplique". La vérité, alors, a un autre sense. Le "francparler", assez écarté de l' "ethos intelectuel" platonicien, y est particulièrement distingué. La parrhesia cynique enracine sa singularité dans une vie autre.

Mots-clés: Vérité. Valeurs. Résistance. Platonisme. Vie cynique. 


\section{Abstract}

To synthesize and develop a series of theoretical propositions from the cynics, worked by Foucault in the course Le courage de la vérité, 1984, is the aim of this article. It distinguishes four general "values" in Greek thought: non-stealth, simplicity, righteousness and immutability - values that assumed different features in the three traditional areas of reflection: logos, eros, bios. But another division, formed in the third area, alêthês bios, deserved more attention of the philosopher. This is where two very different directions of the truth are observed. On the one hand, the philosophical life including specially Platonists, Epicureans and Stoics. On the other, the cynical "existence" "replica of that". The Truth, here, has another meaning. Special distinction is deserved to the "frank speech", very far from the platonic "intellectual ethos". The cynical parrhesia establishes its uniqueness in a life other.

Keywords: Truth. Values. Resistance. Platonism. Cynical life.

\section{Resumo}

Sintetizar e desenvolver uma série de proposições teóricas a partir dos cínicos, trabalhadas por Foucault no curso Le courage de la vérité, de 1984, éo objetivo deste artigo. Destacam-se quatro "valores" gerais do pensamento grego: não dissimulação, simplicidade, retidão e imutabilidade. Valores que assumiram feições diferentes nos três domínios tradicionais de reflexão: logos, eros, bios. Mas uma outra divisão, formada no terceiro domínio, alêthês bios, mereceu maior atenção do filósofo. É aí que se observam dois destinos muito diferentes da verdade. De um lado, a vida filosófica incluindo platônicos, epicuristas e estoicos, principalmente; de outro, a "existência" cínica, "réplica daquela". Verdade, aqui, tem outro sentido. Destaque especial merece o "franco falar", muito distante do "ethos intelectual" platônico. A parrhesia cínica enraíza sua singularidade em uma outra vida.

Palavras-chave: Verdade. Valores. Resistência. Platonismo. Vida cínica.

\section{L'introduction}

J'aimerais évoquer ici quelques éléments proposés par Foucault dans son dernier cours prononcé au Collège de France, le cours de 1984 
intitulé "Le courage de la vérité", plus particulièrement ce qu'il dit à propos des cyniques (FOUCAULT, 2009). Je voudrais reprendre, mais aussi prolonger un certain nombre de thèmes. Il me semble en effet que la richesse des cours au Collège de France de Michel Foucault tient en effet à leur caractère largement programmatique. Il s'agit, comme il le disait en 1982, de proposer des énoncés qui sont "un peu moins que des thèses, mais un peu plus que des hypothèses" (FOUCAULT, 2001). Foucault lance donc une série de propositions théoriques à partir des cyniques qu'il me semble intéressant à la fois de systématiser aussi de prolonger.

Je partirai de ce que Foucault donne à penser, de manière très générale, comme étant les quatre "valeurs" ou "significations" de la vérité pour la pensée grecque (il ne parle pas de critères) (FOUCAULT, 2009). ${ }^{1}$ Il s'agit, successivement de : la non-dissimulation, l'absence de mélanges, la rectitude et l'immuabilité. La vérité, c'est donc ce qui n'est pas caché, ce qui est pur, ce qui est droit et ce qui est constant.

Dans le manuscrit qui servait à la préparation des cours, Foucault avait énoncé un cinquième caractère: l'identité à soi. La vérité, c'est ce qui est de l'ordre du Même. Mais cette caractérisation est finalement biffée sur le manuscrit, et, quand il reprend ces significations assez systématiquement pour décrire ce que serait, classiquement (à savoir: dans les textes de Platon) le vrai amour ou la vraie vie, il continue à barrer dans la suite du manuscrit cette mention de l'identique. Je reviendrai un peu plus tard sur le sens de cet abandon.

La démonstration générale consiste, dans un premier temps, à retrouver, comme je l'ai dit, d'abord dans les textes platoniciens ce que serait une "vraie vie", au sens d'une vie: non-dissimulée, pure, droite et souveraine; puis, dans un second temps, à décrire la subversion cynique, au sens où la vie non cachée du cynique va signifier une existence éhontée, sa vie sans mélange sera une vie de pauvreté et d'infamie, sa vie conforme sera une existence bestiale, et sa vie souveraine une monarchie dérisoire et militante. Voilà pour le mouvement de la démonstration, dans son dessin le plus général.

Leçon du 7 mars 1984. 
Je reviens d'abord sur ces quatre caractères, pour les préciser davantage. Le principe de non-dissimulation, c'est l'idée que le vrai peut et doit s'exposer dans sa nudité, sans masque et sans fards. Le principe de pureté, c'est l'idée que le vrai ne doit pas être mêlé à des éléments étrangers qui risqueraient d'introduire de la fausseté ou de la méprise. Le principe de rectitude, c'est l'idée que le vrai est conforme à des lois, à des régularités. Et enfin le principe de souveraineté, c'est l'idée que le vrai, parce qu'il tient en et par lui-même, demeure.

Alors je crois qu'ici, il est tout à fait envisageable - ce que ne fait pas Foucault directement, mais dont il évoque la possibilité, de montrer que l'application de ces valeurs de vérité au logos, elle nous livre ce que précisément on peut maintenant appeler quatre grands critères de l'alêthês logos, du discours vrai. Le premier critère, c'est la clarté: le discours vrai est clair en ceci que rien en lui ne recèle autre chose que ce qu'il montre, il ne dissimule rien. Le deuxième serait la simplicité: le discours vrai est simple, c'est-à-dire qu'il n'est pas embarrassé par des brillances rhétoriques qui en altèreraient la signification, il est limpide et sans mélange, il n'est troublé par rien. Le troisième critère, c'est la rigueur démonstrative: le discours vrai est entièrement conforme à des règles logiques et déductives. Et enfin, on trouve le critère de nécessité: le discours vrai ne varie pas, il demeure, il est immuable. Clarté, simplicité, rigueur et nécessité: on trouve bien là sans doute ce qu'effectivement on peut appeler les quatre grands critères de vérité du logos.

On peut faire ici deux remarques importantes, et qui nous serviront par la suite. Première remarque: ce logos clair, évident, démonstratif et nécessaire, c'est un logos dont la forme générale est l'identité à soi. Par là, je veux dire que l'identité alors serait moins - ce qui explique les biffures du manuscrit - une valeur de vérité parmi d'autres, que le style propre à la vérité quand elle s'applique à, et détermine le logos.

Ma seconde remarque va concerner cette fois, non pas la forme générale des critères de vérité, mais la forme générale des valeurs de vérité telles que Foucault les a ressaisies et qu'elles peuvent s'appliquer, au logos mais aussi à d'autres domaines comme l'erôs ou le bios. Je veux parler ici de la résistance. En effet, l'alêthês logos par exemple, c'est un discours qui, par sa clarté, résiste à l'exposition - "résister" ici au sens, 
non pas de: s'opposer ou refuser, mais de: passer l'épreuve avec succès et tenir bon -: il ne craint pas de révéler ses significations [et on peut penser là à ce que Foucault en 1983 définissait comme le logos etumos à partir du Phèdre (FOUCAULT, 2008)²]. Ce discours vrai résiste encore à une expression simple, pauvre et sans fioritures (on peut penser ici à l'insistance de Foucault, toujours en 1983, sur la caractérisation par Socrate de son discours dans l'Apologie face à celui de ses adversaires: ma parole est simple, sans rhétorique, parce que je ne sais dire que la vérité). L'alêthês logos résiste encore à sa confrontation avec les règles logiques et autres normes démonstratives. Et il résiste enfin, ce discours vrai, aux vicissitudes du changement et à la corruption.

Ici sans doute il faudrait parler, pour marquer que l'horizon n'est pas proprement politique, d'une "résistance eidétique". Et j'emploierai cette expression d'autant plus volontiers qu'elle permet de renouer la seconde avec la première remarque. Je veux dire que si l'alêthês logos résiste à l'exposition, à la simplicité de l'expression, à la logique et au changement, c'est qu'il a pour objet et fondement les essences, les Formes intelligibles, et que sa résistance n'est que le reflet alors en lui de l'identité parfaite des Idées.

Alors il faudra évidemment se demander, puisque la résistance est la forme générale des valeurs de vérité et qu'elle s'exprime comme résistance du Même quand ces valeurs informent le logos, quelle autre résistance va jouer quand les cyniques poseront le problème de l'alêthês bios, de la vraie vie, cette vraie vie dont Foucault, à propos des cyniques, dira qu'elle est une "vie autre". Auparavant, il faut, je crois, parce que Foucault lui-même en fait le portrait, sommairement au moins évoquer le bios philosophikos, c'est-à-dire la vraie vie en tant qu'elle se construit comme conséquence, déduction, témoignage, attestation de ce logos alêthês. Ce qui m'importe en tout cas, à travers ces expressions un peu vagues (conséquence, déduction, etc.), c'est de considérer ce bios

2 Leçon du 2 mars 1983. 
philosophikos comme dépendant du logos alêthês, puisque je crois que c'est un autre trajet qu'on trouvera avec les cyniques, où on ira du bios alêthinos (la vraie vie) à la parrêsia.

Je caractériserai ce bios philosophikos de manière tout fait sommaire comme une vie "digne", même si ce terme n'est pas employé dans le cours, mais il répond bien, par sa généralité, à nos quatre déterminations, ainsi qu'au style général de l'identité: une vie d'abord honnête, en ce sens surtout qu'elle s'expose sans cesse à son propre jugement (ici Foucault prend l'exemple d'Epictète et de son démon intérieur) et ne dissimule pas des vices cachés sous une respectabilité extérieure, une existence qui ne cache rien, qui n'a pas de replis sombres; au sens deuxièmement d'une certaine austérité, puisque la vie philosophique renonce à la poursuite effrénée des richesses, des plaisirs et des honneurs (la vie sans mélange peut aussi bien renvoyer, précise Foucault, au détachement platonicien qu'à l'autosuffisance stoïcienne ou à la simplicité épicurienne); au sens troisièmement d'une vie qui se soumet aux lois de la cité et aux convenances sociales (et on aurait ici le fameux paradigme de l'obéissance socratique dans le Criton, mais aussi la respectabilité du sage stoïcien), et enfin une vie qui cultive l'indépendance et la maîtrise heureuse de soi.

Je n'insiste pas trop sur ce portrait un peu fade du philosophe et réellement trop général, je voudrais juste souligner à quel point Foucault n'hésite pas à superposer ici des bioi philosophikoi platoniciens, épicuriens et stoïciens, pour les opposer en bloc au bios kunikos, à l'existence cynique. On trouve là un déplacement considérable par rapport à une tendance de fond du cours de 1982 (FOUCAULT, 2001) qui opposait assez facilement le platonisme et le stoïcisme (d'un côté un souci de soi qui prenait la forme d'une vision des essences; et de l'autre un souci de soi attaché à la construction éthique d'un soi fort). Dans les dernières leçons de 1984, Foucault semble beaucoup plus attaché à l'élaboration d'un clivage extrêmement déséquilibré entre le bios $k u$ nikos d'un côté, et de l'autre un bios philosophikos embrassant à la fois le platonisme, l'épicurisme et le stoïcisme. On peut proposer de ce clivage l'explication suivante. Qu'il s'agisse en effet du sage platonicien qui s'attache à fixer les yeux de son esprit à l'éternité des Idées (ou encore 
travaille à instaurer en soi la juste harmonie de ses puissances), du sage épicurien "tel un dieu parmi les hommes" dont la sérénité est aussi douce qu'impeccable, du sage stoïcien conquérant, à force d'exercices, une ataraxia, une tranquillitas imperméables aux agressions du monde, ou même encore de Socrate qui a su, selon l'expression du Lachès que Foucault commente abondamment, faire vibrer entre ses discours et ses actes une consonance parfaite, eh bien toutes ces figures sont et demeurent des figures de l'identité, c'est-à-dire d'une existence ordonnée et harmonieuse. Alors cette identité est diversement, selon les écoles et les textes, dépendante d'un alêthês logos, mais elle demeure le caractère dominant d'un bios philosophikos, contre lequel Foucault pourra précisément faire valoir la discordance dérangeante ou le désordre grimaçant du bios kunikos.

Il faut donc maintenant faire valoir ce que Foucault va construire comme la grande réplique cynique. Très clairement, Foucault explique que les cyniques vont appliquer directement au bios nos quatre valeurs de vérité (FOUCAULT, 2009). ${ }^{3}$ Ici, je ne peux que renvoyer aux leçons du mois de mars 1984, tellement elles sont nourries d'exemples truculents et d'anecdotes absolument farcesques. Je ne retiens là que le mouvement général. La vie des cyniques est non-dissimulée, au sens où ils refusent comme dérisoire jusqu'à la distinction du public et du privé et exposent aux yeux de tous tout à la fois leurs indignations, leur sexualité, leurs dégoûts, leurs dénonciations, etc. Et leur vie est sans mélange, mais parce qu'ils poursuivent agressivement un dénuement qu'ils tentent de porter à sa dernière extrémité, parce qu'ils traquent tout ce qui pourrait faire obstacle à une complète pauvreté. Ils se soumettent bien à la rectitude des lois, mais en suivant à la lettre les prescriptions de la Nature, parce que les convenances humaines sont décidément trop flottantes, et ils préfèrent alors s'inspirer pour vivre du comportement des bêtes. Enfin, leur existence est souveraine, parce que leur combat est sans limites. Alors ce dernier passage est, je crois, un peu plus délicat que les autres et mérite une explicitation supplémentaire. On comprend bien les autres passages à la limite: l'existence non-dissimulée

3 Leçons du 14 et 21 mars 1984. 
comme vie éhontée; la vie sans mélange comme pauvreté radicale; la vie droite comme vie animale. La dernière valeur de vérité est celle de la souveraineté au sens de ce qui ne dépend que de soi. Or le cynique est souverain, pas seulement en ce premier sens obvie, que les monarques demeurent finalement dépendants des contingences historiques, alors que le cynique n'a besoin de rien, mais au sens aussi où leur souveraineté est militante. Ce qui veut dire deux choses: d'abord, que le vrai roi, n'est pas exactement le souverain entouré de sa cour, mais cet athlète de l'existence, ce héros philosophique pour reprendre les expressions employées par Foucault; ensuite, que ce qui fonde la royauté authentique, c'est le combat, mais pas comme conquête de territoires ou victoires militaires, mais plutôt comme posture fondamentale : une manière agressive d'être au service des autres, en les mordant sans cesse par des provocations. Donc la vraie vie du cynique, c'est la constance d'un combat sans limites et la royauté d'une souveraineté totalement indépendante.

Nudité donc du cynique, ainsi que crudité, bestialité, et pour finir ce que Foucault appelle la " militance" du cynique. Et c'est à ce point qu'apparaît le thème, pour finir de l'appel politique à la transformation du monde [le grand "politeuesthai" du cynique pour reprendre l'expression d'Epictète sur laquelle Foucault s'arrête (FOUCAULT, 2009)]. Mais avant d'explorer ce point, je voudrais remarquer trois choses.

Premièrement, on comprend à quel point ce qui est mis en œuvre là, c'est bien encore une résistance comme forme générale de la vérité, mais sur un mode évidemment profondément différent de celui qu'on avait tout à l'heure évoqué. On avait pu dire précédemment: le logos alêthês résiste. Il ne cède pas aux impostures, aux artifices, aux paralogismes, aux variations.

Or la question que pose le cynique à sa propre vie, plutôt qu'à sa pensée, c'est bien: qu'est-ce qui en toi résiste ? Pour rendre peutêtre les choses plus parlantes, on peut sortir un instant de la pensée antique. Après tout, dans le doute cartésien, il s'agit bien d'atteindre une vérité qui absolument résiste, en procédant par étapes: je peux 
douter de l'apparence des choses que je vois ou que je sens parce que j'ai déjà été trompé par mes sens; je peux douter de ma présence effective au monde parce que j'ai pu rêver déjà me trouver dans une situation semblable, alors que j'étais couché au fond de mon lit, etc. Et on sait que Descartes finalement parvient au cogito qui, lui, absolument résiste. Second exemple: dans la méthode phénoménologique, il s'agit, à travers ce qu'on appelle les "variations eidétiques", de dégager des essences en les mettant à l'épreuve de tel ou tel changement de perspective, afin de voir ce qui absolument résiste, et définit alors un invariant. Dans ces exercices où on se dirige vers une "résistance eidétique", le mouvement de la vérité consiste à poser à la pensée le problème de savoir ce qui, de ses contenus, peut résister à un examen poussé.

Or il me semble que Foucault comprend la démarche des cyniques à travers la mise en œuvre de ce qu'il faudrait appeler maintenant une résistance ascétique, passant ainsi, pour reprendre un titre spinoziste, d'une emendatio intellectus à une emendatio vitae. Est-ce que j'ai besoin pour boire d'une coupe en cristal ? Un gobelet en bois y suffit bien. Mais quand Diogène aperçoit au bord d'une fontaine un enfant qui se sert de la paume de ses mains pour boire, il se dépêche de se débarrasser de son pauvre gobelet, car il fait l'épreuve de sa futilité, de sa non-nécessité absolue. De la même manière, en acceptant de coucher par terre, il démontre l'inutilité d'un lit. Ou encore, en se couvrant de son manteau (ou en s'abritant dans son tonneau célèbre), il met en évidence le caractère superflu d'un toit. Ce sont là autant de variations ascétiques permettant de dégager le niveau, évidemment tout à fait rustique, d'un absolument nécessaire.

Mais il faut voir ce qui est atteint par là. A force de gratter l'existence jusqu'à l'os, à force de décaper sa vie de toutes les conventions artificielles ou richesses encombrantes, le cynique parvient véritablement à un élémentaire qui absolument résiste. C'est la fameuse exclamation cynique que rapporte Epictète:

regardez-moi, je n'ai pas de maison, je n'ai pas de patrie, je n'ai pas de richesses et pas de serviteurs. Je n'ai ni femme, ni enfants, ni vaste demeure, mais la terre seule, et le ciel, et un vieux manteau. Mais qu'est-ce 
qui me manque ? Ne suis-je pas sans tristesse, sans peur, ne suis-je pas libre enfin?" (EPICTÈTE, Entretiens, III, 22, 1963, p. 47-48).

Cet élémentaire s'oppose à l'essentiel. Je veux dire que si le philosophe classique met à l'épreuve sa pensée afin d'atteindre une vérité essentielle qui résiste absolument, et pour cela doit traverser le rideau des apparences mouvantes et changeantes, le cynique, lui, creuse l'immanence même de sa vie jusqu'à y conquérir un élémentaire qui serait comme la couche première, solide et dure de l'immanence. Mais parvenant à cet élémentaire, il y puise une énergie sans limites. Ici on pourrait retrouver de nombreux textes d'Epictète dans les Entretiens, dans lesquels le maître stoïcien vante la santé éclatante, solaire, insolente de Diogène. De la même manière, pour reprendre un exemple déjà cité, dont l'illumination du cogito met le feu à la pensée cartésienne, qui dans la foulée peut démontrer la substance éternelle de l'âme, l'existence de Dieu, la vérité des mathématiques et la consistance du monde physique, ainsi le cynique puise, dans l'élémentaire, une force immense qui lui permet de poursuivre indéfiniment son combat et ses luttes.

La seconde grande remarque est que cette vraie vie, comme conquête d'un élémentaire qui résiste, se manifestera par un certain parler-vrai, la fameuse parrêsia cynique - que Foucault prend du reste bien soin de distinguer à la fois de la parrêsia politique (celle d'un Démosthène à l'Assemblée ou d'un Platon face au tyran de Syracuse) et de la parrêsia socratique, quand il agaçait ses concitoyens par ses questions. Cette parrêsia cynique, ce dire-vrai, Foucault dit bien qu'il n'est pas la condition du mode de vie - ce n'est précisément pas un discours de vérité en tant qu'il fonderait, ordonnerait, nourrirait un mode de vie -, mais plutôt sa conséquence, ou en tout cas sa dramatisation verbale: de la même manière que la vie cynique fait valoir, dans la trame visible de son existence, la nudité, la pauvreté, la bestialité et l'agressivité du vrai, ces mêmes valeurs sont remises en scène dans une parole qui dénonce l'hypocrisie des convenances, la vanité des richesses, l'artificialité des codes sociaux, et la dérision des indépendances. En tous les cas, on comprend bien qu'alors que le logos alêthês tentait de débrouiller le maquis, de traverser le brouillard 
de la doxa, des erreurs, des opinions reçues, des idées toutes faites, afin d'atteindre des vérités pures, la parrêsia, elle, écarte violemment la broussaille des conventions artificielles, des habitudes sociales, des normes culturelles, pour dégager un élémentaire radical.

La troisième et dernière remarque concerne ce que Foucault désigne comme le "renversement cynique de la vraie vie en vie autre". Le bios kunikos en effet ne fait rien d'autre que d'appliquer à la vie même les valeurs de vérité, mais avec une radicalité telle qu'elle devient un objet de scandale. Foucault insiste particulièrement sur ce point : la vraie vie du cynique est une existence scandaleusement dissonante. Ici on retrouverait bien sûr la grande alternative à ce que j’avais désigné comme le style général d'identité qui marquait le moment où les valeurs de vérité devenaient des critères de vérité de l'alêthês logos (discours vrai) et informaient un bios philosophikos recueilli et harmonieux. Avec les cyniques, c'est plutôt un style général d'altérité qui se dégage de l'application directe et radicale des valeurs de vérité au bios. D'abord parce que cette vie scandaleusement détonne; ensuite parce qu'elle nourrit une parrêsia qui ne cesse de dénoncer une hypocrisie et une corruption généralisées, et arrache donc des masques de respectabilité qui cachent des comportements contraires aux principes affichés. Ce mouvement-là est important: l'altérité du bios kunikos doit faire éclater le mensonge des comédies sociales. Il s'agit donc moins de faire signe vers des identités que de démasquer des contradictions.

$* * *$

En reprenant ici l'insistance de Foucault sur le caractère scandaleux, provocateur, dissonant du bios kunikos, on pourrait dire que cette vie produit de "l'inacceptable". Foucault n'introduit pas directement cette notion, mais je pense qu'il pourrait être intéressant de la construire. Je ne veux pas l'entendre ici au sens où par exemple Boltanski à propos de Bourdieu l'emploie quand il dit: la sociologie, c'est ce qui doit rendre la réalité inacceptable, c'est-à-dire précisément dénoncer les systèmes sociaux comme autant de machines destinées à rendre acceptable l'inacceptable (et l'inacceptable ici va désigner: des injustices 
sociales, des confiscations politiques, etc.). L'inacceptable cynique, c'est plutôt ce qui constitue par rapport aux normes existantes, aux conventions reçues, aux vérités partagées, une provocation. Mais - et Foucault insiste particulièrement sur ce point - il ne s'agit pas ici de jouer la transgression pour faire briller l'éclat d'une marginalité triomphante. L'inacceptable cynique (leur vie, leurs comportements, leurs prises de parole, leurs attitudes) ne vaut que comme adresse, mise en demeure par lesquelles chacun est amené à faire l'épreuve de ses propres contradictions. Il s'agit de dire des choses inacceptables, mais au sens où d'une certaine manière elles sont trop vraies, et que la parrêsia pousse l'affirmation de vérité au-delà de toute décence. Ici, on pourrait faire le lien je crois avec le cours prononcé en 1983, à propos de la parrêsia démocratique (FOUCAULT, 2008). ${ }^{4}$ Quand Foucault décrit une prise de parole authentiquement démocratique, c'est pour montrer qu'elle consiste moins à trouver un consensus, à pacifier les différends, qu'à briser la mise en commun des lâchetés et le confort des majorités silencieuses. Parler vrai, c'est obliger chacun à reconnaître qu'il est moins d'accord avec lui-même et avec les autres qu'il ne l'admet lui-même. En ce sens la vérité est inacceptable, si elle désigne non pas ce qui réconcilie chacun avec lui-même, mais ce mouvement par lequel précisément chacun est appelé à se transformer et ne pas se reposer dans l'illusion confortable d'une identité définitive et stable. Enfin l'inacceptable met en jeu une énergie de transformation. Cette idée de transformation est bien indiquée par Foucault quand il explique que la vie scandaleusement autre du cynique est un appel à la transformation de soi et du monde (ce qu'il nomme le "politeuesthai" des cyniques, la grande politique cynique). Ce qui est inacceptable, c'est le Même, c'est-à-dire la perpétuation indéfinie des hypocrisies ou des compromissions.

Tout ceci nous amène à une distinction fondamentale, sur laquelle je conclurai, entre deux figures opposées de l' "Autre" - ce qu'indique bien Foucault à la fin du cours quand il oppose le platonisme et le cynisme (FOUCAULT, 2009). ${ }^{5}$ Il y aurait 1'“autre monde" des métaphysiques

4 Leçons du 2 et du 9 février 1983.

5 Leçon du 28 mars 1984 (cf. surtout p. 310-311 le passage cité du manuscrit). 
platoniciennes: l'au-delà des essences intelligibles, le monde séparé des essences et des vérités éternelles (qui est autre en tant que séparé de l'immanence sensible, mais identique en soi). Et puis il y a le "monde autre", au sens de l'immanence transformable par les énergies politiques, l'appel à cette transformation étant constituée par la vie scandaleusement autre du cynique qui interpelle, bouscule et dérange. Il y a l' "autre vie" des platoniciens: la vie rêvée d'un au-delà débarrassé des contingences matérielles, et il y a la "vie autre" des cyniques: cette existence dérangeante qui fait éclater, au cœur de la cité, le scandale de la vérité.

\begin{tabular}{|c|c|c|c|c|}
\hline LE VRAI & $\begin{array}{l}\text { LOGOS } \\
\text { ALÊTHÊS }\end{array}$ & $\begin{array}{l}\text { BIOS } \\
\text { PHILOSOPHIKOS }\end{array}$ & $\begin{array}{l}\text { BIOS } \\
\text { KUNIKOS }\end{array}$ & PARRÊSIA \\
\hline Non-caché & Evidence & Honnêteté & Nudité & $\begin{array}{l}\text { Contre } \\
\text { hypocrisies }\end{array}$ \\
\hline Pur & Clarté & Austérité & Pauvreté & $\begin{array}{l}\text { Contre } \\
\text { Compromissions }\end{array}$ \\
\hline Droit & Rigueur & $\begin{array}{l}\text { Conformisme et } \\
\text { Décence }\end{array}$ & Animalité & $\begin{array}{l}\text { Contre } \\
\text { Conventions }\end{array}$ \\
\hline Constant & Nécessité & Indépendance & Souveraineté & $\begin{array}{l}\text { Contre } \\
\text { Consensus }\end{array}$ \\
\hline RESISTANCE & \multicolumn{2}{|c|}{ IDENTITE A SOI } & \multicolumn{2}{|c|}{ ALTERITE SCANDALEUSE } \\
\hline Essentiel & Digne & Elémentaire & \multicolumn{2}{|c|}{ Inacceptable } \\
\hline Autre monde & \multicolumn{2}{|c|}{ Autre vie } & atre & Monde autre \\
\hline \multicolumn{3}{|c|}{ TRANSCENDANCE ET RENONCEMENT } & MANENCE ET TF & NSFORMATION \\
\hline
\end{tabular}

\section{Références}

FOUCAULT, M. L'herméneutique du sujet. Cours au Collège de France, 19811982. Éd. Frédéric Gros. Paris: Gallimard; Seuil, 2001. 
FOUCAULT, M. Le gouvernement de soi et des autres. Cours au Collège de France, 1982-1983. Éd. Frédéric Gros. Paris: Gallimard; Seuil, 2008.

FOUCAULT, M. Le courage de la vérité: le gouvernement de soi et des autres II. Cours au Collège de France, 1983-1984. Éd. Frédéric Gros. Paris: Gallimard; Seuil, 2009.

ÉPICTÈTE. Entretiens, III. Traduction de J. Souilhé. Paris: Les Belles Lettres, 1963. p. $47-48$.

Reçus: 09/10/2010

Recebido: 09/10/2010

Approuvé: 08/12/2010 Aprovado: 08/12/2010 